\title{
On Microwave Breast Imaging with Ultrasound Spatial Priors
}

\author{
Nozhan Bayat*, Pedram Mojabi, Joe LoVetri, and Puyan Mojabi \\ Department of Electrical and Computer Engineering, University of Manitoba, Winnipeg, Manitoba, Canada
}

\begin{abstract}
We investigate the use of ultrasound images as prior structural information (also known as ultrasound spatial priors) to guide microwave breast imaging so as to enhance its achievable complex permittivity images. In the main approach considered herein, the edges within the discretized ultrasound compressibility image are fed as spatial priors into a microwave imaging algorithm. It is shown that this method requires minimal post-processing of the ultrasound image and can enhance the achievable microwave image accuracy. It is also demonstrated that small tumours can still go undetected in microwave breast imaging using this method if their edges are missing from the spatial priors.
\end{abstract}

\section{Introduction}

Microwave imaging (MWI) is an imaging modality that aims to create quantitative images of the relative complex permittivity of an object of interest (OI). Recently, to enhance the achievable MWI image accuracy, hybrid imaging approaches have been considered. The main idea has been to use a higher resolution imaging modality such as ultrasound [1, 2] or magnetic resonance imaging [3, 4] to create structural information to be used by MWI algorithms. These structural information are also referred to as spatial priors (SP). In this work, we deal with tomographic microwave breast imaging, and in particular we derive the SP from the reconstructed ultrasound compressibility image. These SP will then be fed into an MWI inversion algorithm so as to observe how much the MWI complex permittivity image can be improved. The important aspects of the method considered are the followings. 1) The derivation of the SP only requires a minimal and simple post-processing of the ultrasound compressibility image; 2) no permittivity assignment to SP is needed; 3) the MWI inversion algorithm starts with a trivial initial guess, and the SP only guide its convergence; 4) the SP can be partial, and does not need to cover the whole domain; 5) If no SP are provided, the algorithm converts back to the regular blind inversion.

\section{Methodology}

There are several reported methods with which SP can be incorporated into MWI algorithms [4-10]. These methods have been classified under four categories in Fig- ure 1. Herein, we consider the first two categories. The first one favours the similarity between complex permittivity within each region of the SP [4--7]. For this category, we use the so-called MRSP-GNI algorithm [6] which uses an extra layer of SP regularization in conjunction with the multiplicative regularized Gauss-Newton inversion (MR-GNI). In the second category, the SP are provided in the form of preferred edges to the inversion algorithm [8]. For this category, we consider the following two microwave imaging algorithms: augmented multiplicative regularized Gauss-Newton inversion (AMR-GNI) and augmented multiplicative regularized contrast source inversion (AMR-CSI). These algorithms, described in [8], augment the standard MR used with MR-GNI and MR-CSI with an extra term which represents the preferred edges. As can be seen in Figure 1, these favoured edges are provided in the form of $|\nabla P|$ where $\nabla$ is the gradient operator and $P$ can be derived from an ultrasound image. The main novelty of this paper compared to [6,8] lies in obtaining the SP from ultrasound imaging 11

\section{Results}

Consider the numerical breast phantom shown in Figure 2 , which includes three tumours. This figure represents the real and imaginary part of the relative complex permittivity of this breast model at $1 \mathrm{GHz}$ as well as its true quantitative ultrasonic compressibility at $100 \mathrm{KHz}$ and its attenuation slope respectively. MWI uses 36 transceivers and ultrasound imaging uses 64 transducers ${ }^{2}$ Before evaluating the use of ultrasound-derived SP, let us begin by considering the complete and perfect SP. The perfect SP for favouring the similarity between the complex permittivity in each region are shown in Figure 3 (a) whereas the perfect SP for favouring edges are shown in Figure 3 (b). The reconstruction results obtained by the MRSP-GNI and AMR-GNI algorithms have been shown in Figures 3(c)-(f) ${ }^{3}$ As can be seen, both of these algorithms are relatively successful. In the next step, we consider a more practical case where

\footnotetext{
${ }^{1}$ We note that instead of using ultrasound images as SP in MWI inversion, we can simultaneously reconstruct both ultrasound and microwave properties using appropriate MR, e.g., the cross gradient MR [11] or using the gradient of the ultrasound property within the microwave MR and using the gradient of the microwave property within the ultrasound MR [12].

${ }^{2}$ MWI and ultrasound data are contaminated with $3 \%$ and $5 \%$ noise.

${ }^{3}$ The reconstruction results of the AMR-CSI algorithm have not been shown as they were not as successful as the AMR-GNI reconstructions.
} 


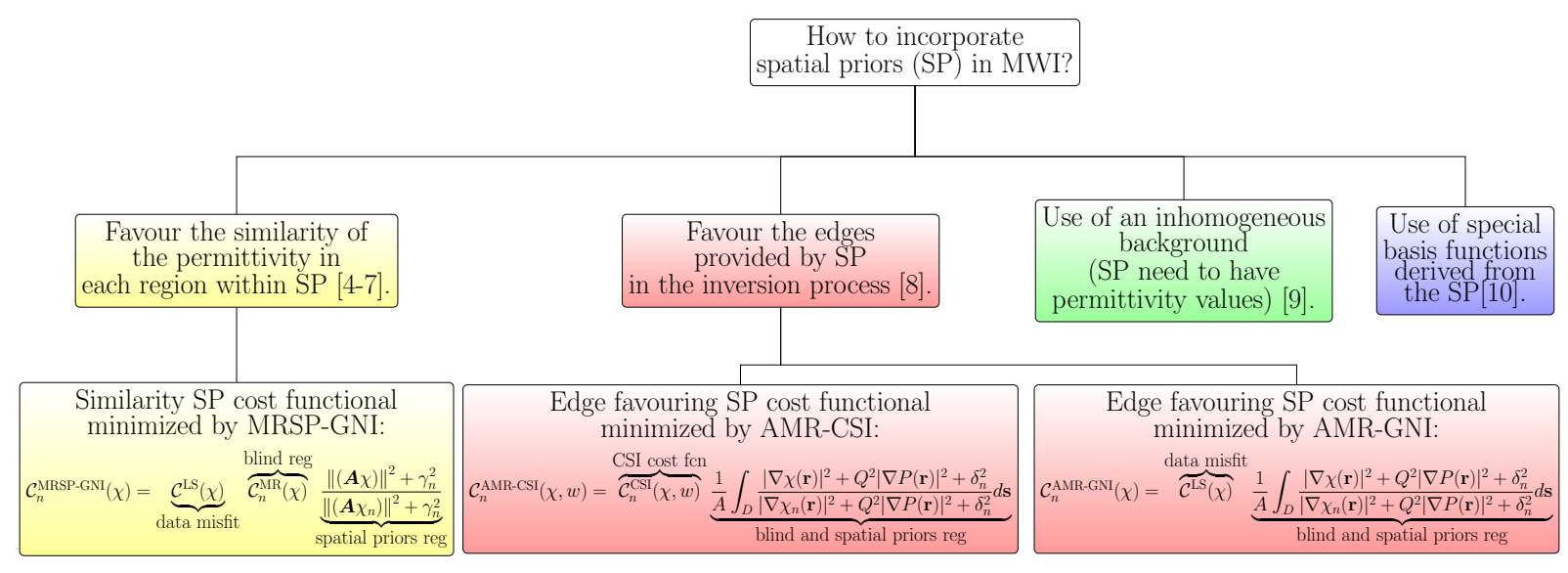

Figure 1. Four different categories of methods with which prior structural information, also known as spatial priors (SP), can be incorporated into microwave imaging (MWI) inversion algorithms. The focus of this paper is on three MWI inversion algorithms equipped with SP, namely, MRSP-GNI, AMR-GNI and AMR-CSI. (For AMR-CSI and AMR-GNI, $Q=10$.)

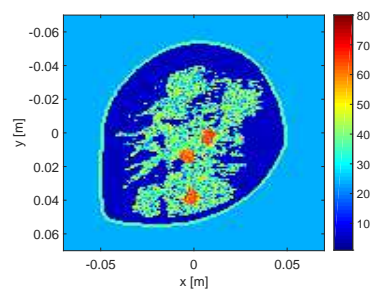

(a) $\operatorname{True} \operatorname{Re}\left(\varepsilon_{r}\right)$

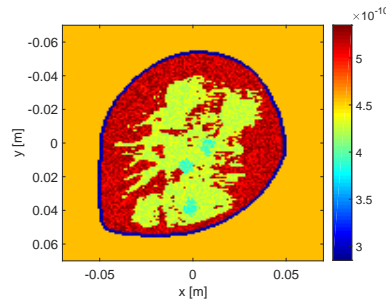

(c) True Compressibility $(\kappa)$

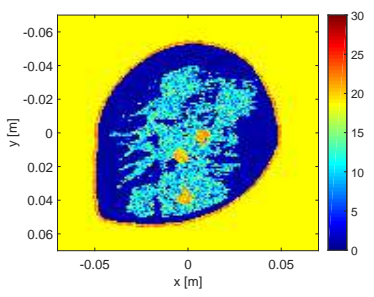

(b) True $-\operatorname{Im}\left(\varepsilon_{r}\right)$

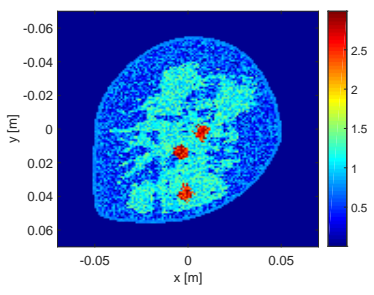

(d) True Attenuation slope $(\alpha)$

Figure 2. Numerical breast phantom. (Top) Real and imaginary parts of the relative complex permittivity. (Bottom) Ultrasonic compressibility and attenuation slope.

the SP (favouring edges) have been derived from the ultrasound compressibility image. As shown in Figure 4, the compressibility image is first reconstructed by inverting the ultrasound data. This compressibility image is then given to the MATLAB function discretize to generate a matrix $P$ with four discretized bins. The absolute of the gradient of the matrix $P$, which represents the edges in the ultrasound image, are then provided as the SP to MWI. The AMRCSI and AMR-GNI reconstructions which utilize the ultrasound SP are capable of reconstructing the tumours with the AMR-GNI outperforming AMR-CSI in resolving all the three tumours. It is also worthwhile noting the blind inversion using the MR-GNI and MR-CSI algorithms in this fig-

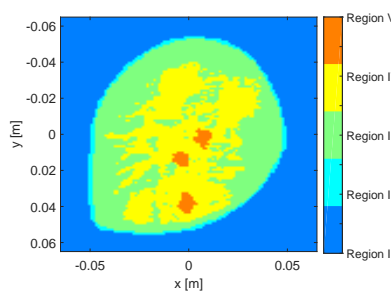

(a) SP (Similarity)

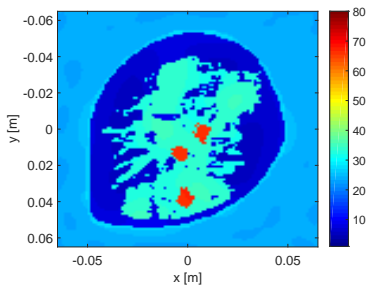

(c) $\operatorname{Re}\left(\varepsilon_{r}\right)$, MRSP-GNI

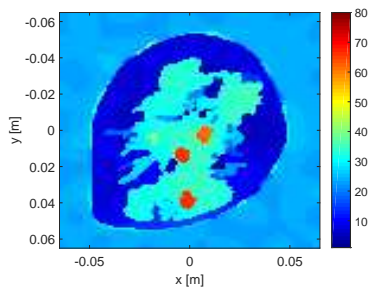

(e) $\operatorname{Re}\left(\varepsilon_{r}\right)$, AMR-GNI

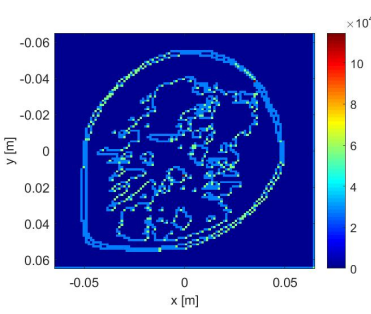

(b) SP (Edge)

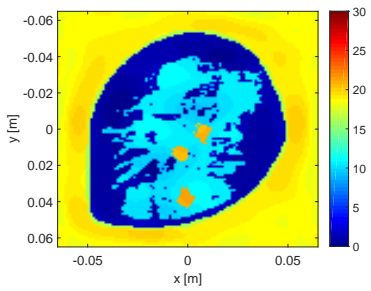

(d) $-\operatorname{Im}\left(\varepsilon_{r}\right)$, MRSP-GNI

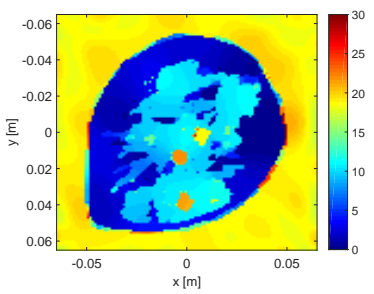

(f) $-\operatorname{Im}\left(\varepsilon_{r}\right)$, AMR-GNI
Figure 3. Perfect SP in the form of favoured (a) similarity in each region and (b) edges. Reconstructed relative complex permittivity using (c)-(d) MRSP-GNI (favoured similarity) and (e)-(f) AMR-GNI (favoured edges).

ure in which the spatial resolution is not sufficient to resolve these tumours. This example demonstrates the promising potential of using SP in the form of preferred edges as it 


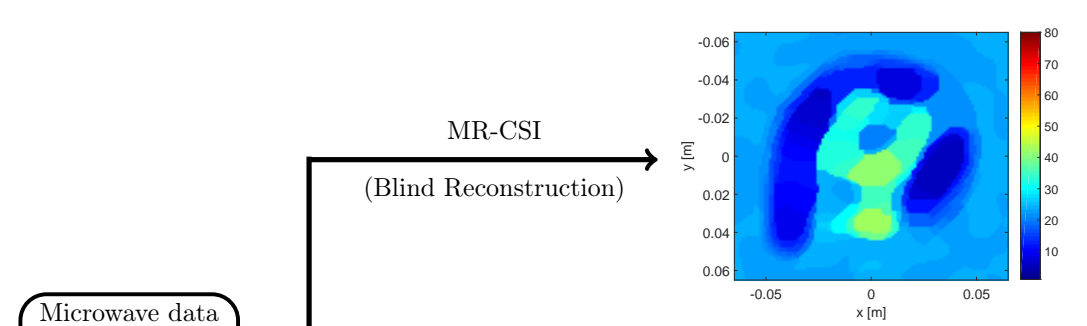

(a) $\operatorname{Re}\left(\epsilon_{r}\right)$

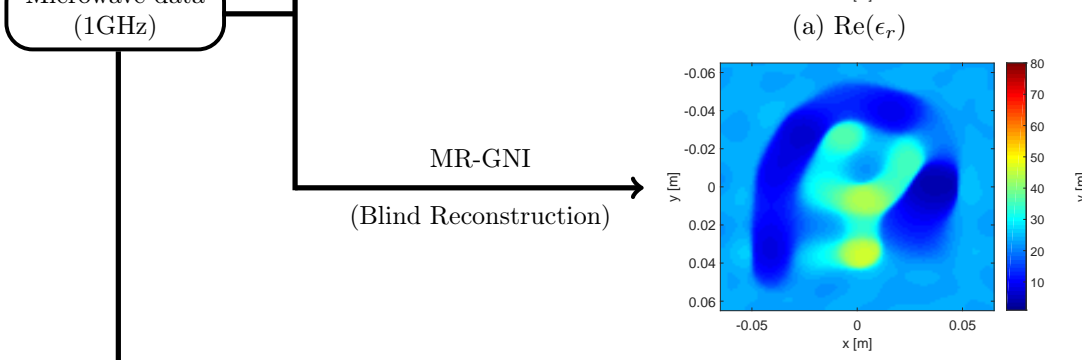

(c) $\operatorname{Re}\left(\epsilon_{r}\right)$

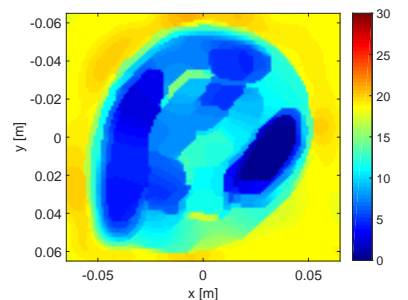

(b) $-\operatorname{Im}\left(\epsilon_{r}\right)$

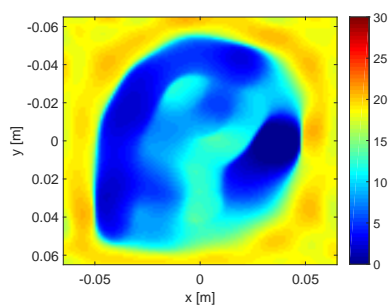

(d) $-\operatorname{Im}\left(\epsilon_{r}\right)$

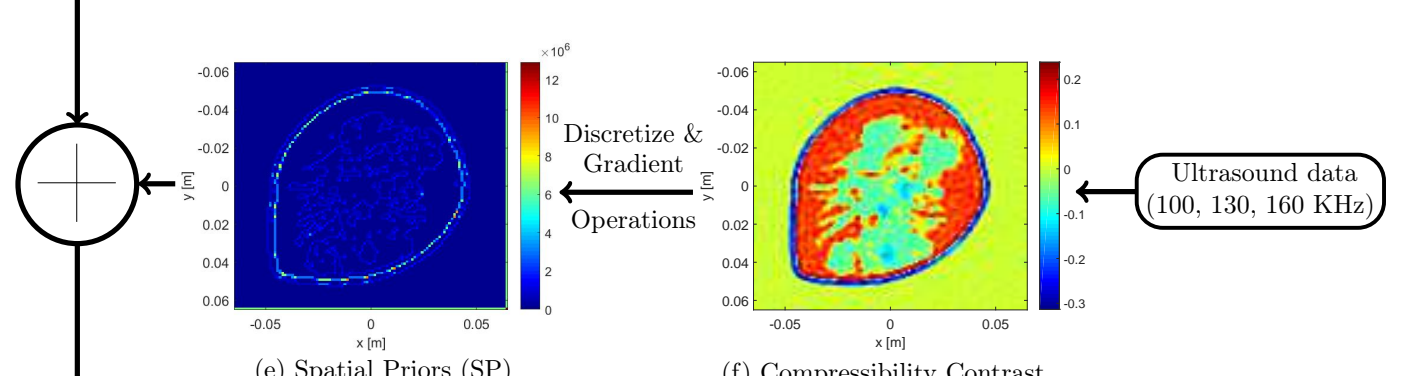

(e) Spatial Priors (SP)

(f) Compressibility Contrast

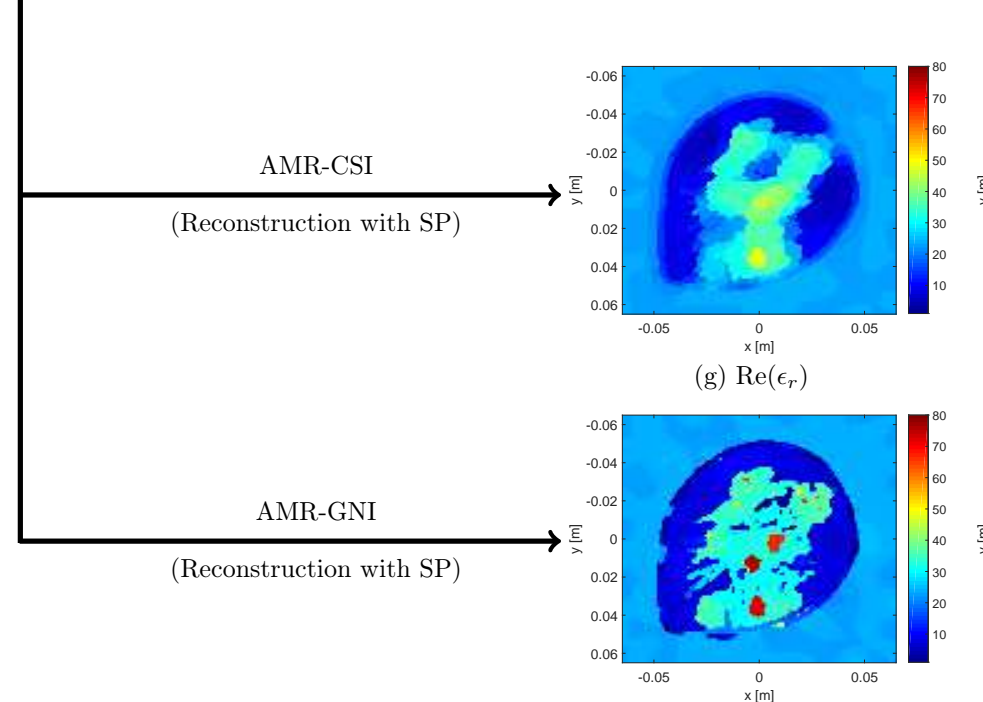

(i) $\operatorname{Re}\left(\epsilon_{r}\right)$

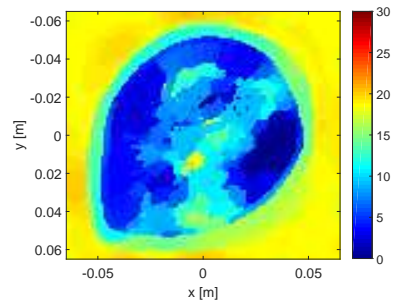

(h) $-\operatorname{Im}\left(\epsilon_{r}\right)$

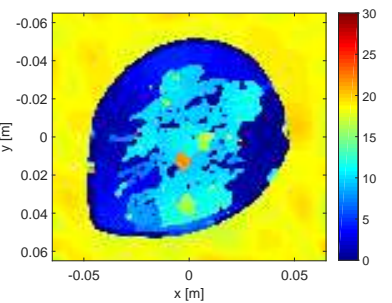

(j) $-\operatorname{Im}\left(\epsilon_{r}\right)$

Figure 4. (First and Second Rows) Blind single-frequency microwave data inversion using the MR-CSI and MR-GNI algorithms. (Third Row) Ultrasound data inversion and preparation of the spatial priors (SP) in the form of favoured edges. (Fourth and Fifth Rows) Microwave data inversion with the ultrasound-derived SP using the AMR-CSI and AMR-GNI algorithms.

only requires a minimal post-processing of ultrasound compressibility images. Finally, let us consider another practical case where the SP (favouring edges) does not include any indications of tumours as shown in Figure 5 . As can be seen, the AMR-GNI algorithm is not able to resolve these three tumours in this case. It should be noted that the diameter of these tumours is about $6 \mathrm{~mm}$ or $\lambda_{b} / 10$ where $\lambda_{b}$ is the microwave wavelength in the background medium.

\section{Conclusions}

We have investigated the use of ultrasound images as prior structural information, referred to as ultrasound spatial priors, for microwave breast imaging. In particular, we have shown that such structural information can be provided in the form of preferred edges to MWI algorithms. The inclusion of prior structural information can enhance the achiev- 


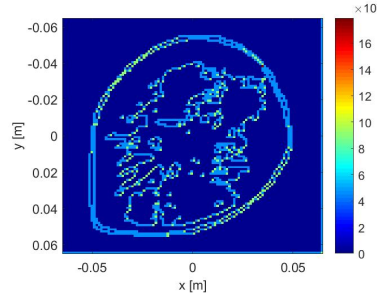

(a) SP (edges) with no tumours

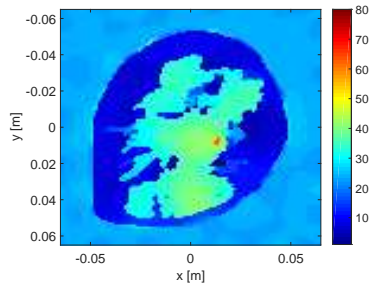

(b) $\operatorname{Re}\left(\varepsilon_{r}\right)$, AMR-GNI

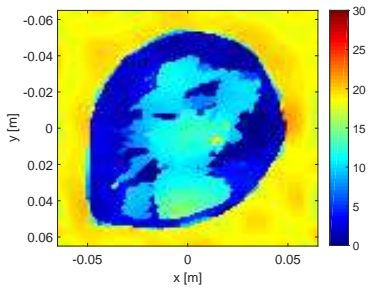

(c) $-\operatorname{Im}\left(\varepsilon_{r}\right)$, AMR-GNI
Figure 5. (a) The perfect SP when the edges associated with the three tumours are removed. (b)-(c) The reconstruction results using the AMR-GNI algorithm.

able MWI image accuracy. Two important observations were also made. 1) The small tumours considered can still go undetected in the MWI image if the provided spatial priors do not include the tumour edges. 2) Once the SP contain the edges of the tumours, the MWI algorithm was able to reconstruct the tumours' relative complex permittivity. (The reconstructed value was about $71-j 16$ for what it should be about $63-j 20$.) These two observations may suggest that the collected microwave data contained information about some inclusions in the fibroglandular tissue; however, the blind microwave imaging algorithm was not able to retrieve the high spatial frequency components required to properly resolve these tumours. Once the SP including the tumour edges are provided, the imaging algorithm has access to high spatial frequency components to represent the tumour regions. These observations are still at the preliminary stage, and we need to perform more studies using different numerical breast phantoms to be able to arrive at a firm conclusion. Finally, the use of ultrasound attenuation image in conjunction with the ultrasound compressibility image will be studied as SP for this application.

\section{Acknowledgements}

We would like to thank Prof. Elise Fear for providing the numerical breast phantom, and the financial support of NSERC and CRC of Canada.

\section{References}

[1] H. Jiang, C. Li, D. Pearlstone, and L. Fajardo, "Ultrasound-guided microwave imaging of breast cancer: tissue phantom and pilot clinical experiments," Med. Phys., vol. 32, 2005.
[2] M. Omer, P. Mojabi, D. Kurrant, J. LoVetri, and E. Fear, "Proof-of-concept of the incorporation of ultrasound-derived structural information into microwave radar imaging," IEEE J. Multiscale Multiphys. Comput. Tech., vol. 3, pp. 129-139, 2018.

[3] G. Boverman, C. Davis, S. Geimer, and P. M. Meaney, "Image registration for microwave tomography of the breast using priors from non-simultaneous previous magnetic resonance images," IEEE Journal of Electromagnetics, RF and Microwaves in Medicine and Biology, vol. PP, no. 99, pp. 1-1, 2017.

[4] A. H. Golnabi, P. M. Meaney, S. D. Geimer, and K. D. Paulsen, "3-D microwave tomography using the soft prior regularization technique: Evaluation in anatomically realistic MRI-derived numerical breast phantoms," IEEE Transactions on Biomedical Engineering, vol. 66, no. 9, pp. 2566-2575, Sep. 2019.

[5] N. Bayat, Advancing Microwave Imaging Algorithms and Techniques, $\mathrm{PhD}$ thesis, University of Manitoba, Manitoba, Canada, 2019 [available online].

[6] N. Bayat and P. Mojabi, "Incorporating spatial priors in microwave imaging via multiplicative regularization," IEEE Transactions on Antennas and Propagation (Early Access), pp. 1-12, 2019.

[7] L. M. Neira, B. D. V. Veen, and S. C. Hagness, "High-resolution microwave breast imaging using a 3D inverse scattering algorithm with a variable-strength spatial prior constraint," IEEE Trans. Antennas and Propag., vol. 65, no. 11, pp. 6002-6014, Nov 2017.

[8] N. Bayat and P. Mojabi, "A multiplicative regularizer augmented with spatial priors for microwave imaging," Submitted to IEEE Transactions on Antennas and Propagation, pp. 1-5, 2020.

[9] N. Abdollahi, D. Kurrant, P. Mojabi, M. Omer, E. Fear, and J. LoVetri, "Incorporation of ultrasonic prior information for improving quantitative microwave imaging of breast," IEEE Journal on Multiscale and Multiphysics Computational Techniques, vol. 4, pp. 98-110, 2019.

[10] M. T. Bevacqua, R. Scapaticci, G. G. Bellizzi, T. Isernia, and L. Crocco, "Permittivity and conductivity estimation of biological scenarios via 3D microwave tomography," in 13th European Conference on Antennas and Propagation (EuCAP), 2019, pp. 1-3.

[11] A. Abubakar, G. Gao, T. M. Habashy, and J. Liu, "Joint inversion approaches for geophysical electromagnetic and elastic full-waveform data," Inverse Problems, vol. 28, no. 5, p. 055016, 2012.

[12] X. Song, M. Li, F. Yang, S. Xu, and A. Abubakar, "Study on joint inversion algorithm of acoustic and electromagnetic data in biomedical imaging," IEEE Journal on Multiscale and Multiphysics Computational Techniques, vol. 4, pp. 2-11, 2019. 